\title{
Optimising the Gaia scanning law for relativity experiments
}

\section{Jos de Bruijne ${ }^{1} \dagger$, Hassan Siddiqui ${ }^{2}$, Uwe Lammers ${ }^{2}$, John Hoar ${ }^{2}$, William O'Mullane ${ }^{2}$, and Timo Prusti ${ }^{1}$}

\footnotetext{
${ }^{1}$ Research and Scientific Support Department of the European Space Agency, European Space Research and Technology Centre, Keplerlaan 1, 2201 AZ, Noordwijk, The Netherlands

${ }^{2}$ Science Operations Department of the European Space Agency, European Space Astronomy Centre, Villanueva de la Cañada, 28692 Madrid, Spain
}

\begin{abstract}
Gaia is ESA's upcoming astrometry mission, building on the heritage of its predecessor, Hipparcos. The Gaia nominal scanning law (NSL) prescribes the ideal attitude of the spacecraft over the operational phase of the mission. As such, it precisely determines when certain areas of the sky are observed. From theoretical considerations on sky-sampling uniformity, it is easy to show that the optimum scanning law for a space astrometry experiment like Gaia is a revolving scan with uniform rotation around the instrument symmetry axis. Since thermal stability requirements for Gaia's payload require the solar aspect angle to be fixed, the optimum parallax resolving power is obtained by letting the spin axis precess around the solar direction. The precession speed has been selected as compromise, limiting the across-scan smearing of images when they transit the focal plane, providing sufficient overlap between successive "great-circle" scans of the fields of view, and guaranteeing overlap of successive precession loops. With this scanning law, with fixed solar-aspect angle, spin rate, and precession speed, only two free parameters remain: the initial spin phase and the initial precession angle, at the start of science operations. Both angles, and in particular the initial precession angle, can be initialized following various (programmatic) criteria. Examples are optimization/fine-tuning of the Earth-pointing angle, of the number and total duration of Galactic-plane scans, or of the ground-station scheduling. This paper explores various criteria, with particular emphasis on the opportunity to optimise the scanning-law initial conditions to "observe" the most favorable passages of bright stars very close to Jupiter's limb. This would allow a unique determination of the light deflection due to the quadrupole component of the gravitational field of this planet.
\end{abstract}

Keywords. relativity, space vehicles: instruments, astrometry, planets and satellites: Jupiter

\section{Jupiter's quadrupole moment}

For Jupiter, the magnitude of the gravitational monopole deflection for a grazing ray is 16 mas. Superimposed on this monopole, there is a quadrupole field with an amplitude of $240 \mu$ as. This quadrupole deflection, which is within Gaia's reach, has a specific pattern which is a function of (i) the position of the star with respect to the oblate deflector, and (ii) the orientation of Jupiter's spin axis. It is, therefore, possible with Gaia to carry out some kind of eclipse experiment - similar to that of Dyson, Eddington, and Davidson in 1920 - by comparing stellar positions in the immediate vicinity of Jupiter (Crosta \& Mignard (2006)). We plan to do so and propose to start Gaia's nominal scanning law after launch with optimum initial conditions, such that the maximum number of passages of bright stars close to Jupiter's limb will be observed. This, in turn, will optimise the end-of-mission detection significance of the quadrupole light deflection.

$\dagger$ This poster describes work which is carried out in the frame of the RElativistic Models And Tests (REMAT) group of the Gaia Data Processing and Analysis Consortium (DPAC). 


\section{The Gaia Nominal Scanning Law (NSL) and operational strategy}

During its 5-year operational life time, Gaia will continuously scan the sky using a uniform, revolving scanning law - the Nominal Scanning Law (NSL) - satisfying the loop-overlap condition. The NSL is determined by fixing five constants and two functions: the inertial spin rate $\omega\left(=60 \operatorname{arcsec} \mathrm{s}^{-1}\right.$; rotation period $\left.=6 \mathrm{~h}\right)$; the revolving angle, also known as solar-aspect angle $\xi\left(=45^{\circ}\right)$; the precession-speed constant $S(=4.223$; 5.8 spin-axis revolutions per year); the initial spin phase $\Omega_{0}$ (free parameter); the initial revolving phase $\nu_{0}$ (free parameter); and the ecliptic coordinates of the nominal Sun $\lambda_{\mathrm{s}}(\mathrm{t}), \beta_{\mathrm{s}}(\mathrm{t})$ (fixed by Nature). The spin-axis coordinates $\lambda_{\mathrm{z}}(\mathrm{t}), \beta_{\mathrm{z}}(\mathrm{t})$ and spin phase $\Omega(\mathrm{t})$ follow from solving a set of coupled differential equations. Since $\omega, \xi$, and $S$ have been fixed for Gaia, the NSL is fully determined once the two free parameters $\left(\Omega_{0}\right.$ and $\left.\nu_{0}\right)$ are defined, which is planned for at the start of the mission. We investigate the optimum values for these free parameters from the mission point of view.

During each 6-hour revolution around its spin axis, each Gaia field of view (FoV) sweeps out a thickened great circle which crosses the Galactic plane twice. The typical 6-hour data-collection rate of the two FoVs combined contains 4 peaks. During specific periods of the mission, the spin axis points close to one of the Galactic poles. Due to the relatively slow precession of the spin axis, such situations can be sustained for several consecutive days. During these periods, the FoVs effectively continuously sweep along the Galactic plane, with high sustained data rates as a result. These periods are known as Galactic-plane scans. Typically, a handful of such periods occur during each year of the mission. When they occur depends on the NSL initial conditions.

\section{Ground-station support}

Gaia observes 24 hours per day. Typically, however, ground-station contact is limited to 8 hours per day as a result of programmatic constraints. During the daily non-visibility periods, collected data are stored on board in a mass memory. Mass-memory saturation resulting in data loss and science-performance degradation - occurs about two dozen times over the mission. These events are associated with Galactic-plane scan periods. In order to minimize data losses, the systematic support of a second ground station is scheduled during Galactic-plane scan periods. When Galactic-plane scan periods precisely occur, depends on the NSL initial conditions, in particular the initial revolving phase $\nu_{0}$. The peak-to-valley variation of the total number of Galactic-plane-scan days is $40 \%$ over all possible initial angles. The NSL initial conditions, thus, determine the total groundstation support required and, hence, are a cost factor.

\section{Ground-station conflicts}

ESA's ground-station network includes two Deep Space Antennae: a 35-m dish in Cebreros (Spain) and a 35-m antenna in New Norcia (Australia). These antennae are shared resources. During Gaia's operational life (2012-2017), at least one conflicting user has been identified, namely Rosetta. The Rosetta mission is an interplanetary mission whose objective is to rendezvous with and make in-situ measurements of comet 67 P/Churyumov-Gerasimenko. These events will take place in summer/autumn 2014. Assuming Rosetta's ground-station needs during this period will be met with high priority, limited station hours will be left for Gaia during summer 2014. From a ground-station conflict analysis, it follows that Gaia Galactic-plane scans should be avoided from June through August 2014. This will require dedicated NSL initial conditions. 


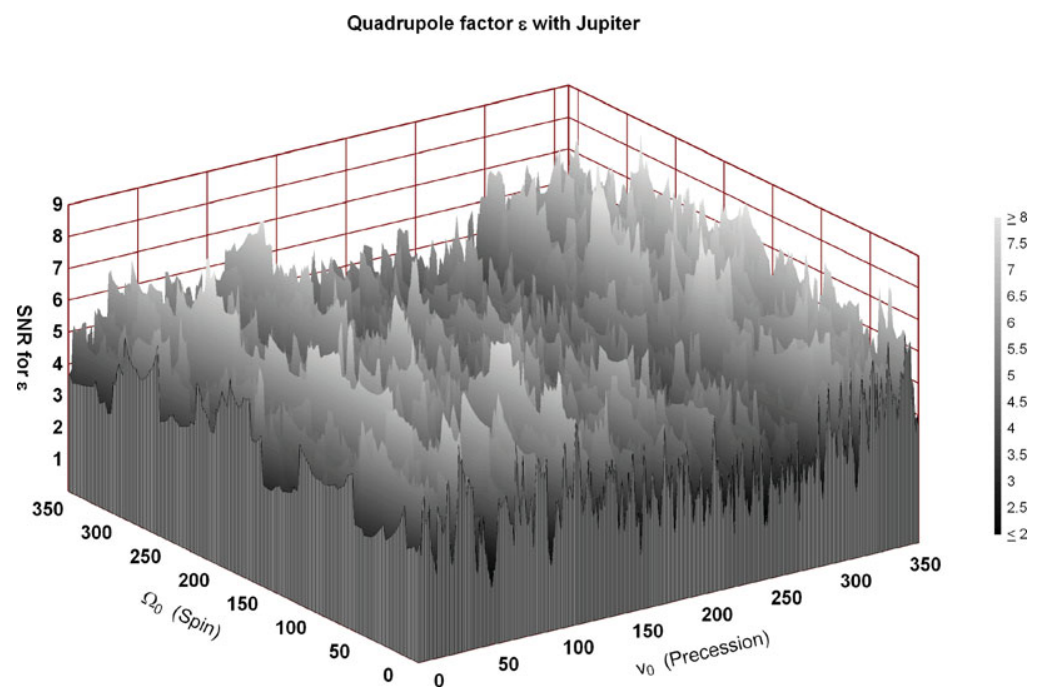

Figure 1. Signal-to-Noise Ratio (SNR) of the detection of the Jupiter quadrupole deflection measurement as function of NSL initial angles. Figure and analysis courtesy François Mignard.

\section{Spin-axis - Earth angle}

Gaia uses its Phased-Array Antenna (PAA) to transmit its data to the ground. Towards the edges of the usable range $\left(15^{\circ}\right.$ away from the central $45^{\circ}$ direction), the PAA looses power. It is, hence, favorable for Gaia's overall data return - through an improved link budget and potentially more efficient convolutional encoding of the telemetry stream to minimise the angle between the spin axis and the Gaia-Earth vector. The evolution of this angle over the mission depends on the NSL initial conditions. Optimised NSL initial conditions can reduce the peak-to-valley variations of the angle by $1^{\circ}$ on both extremes, with favorable consequences for the overall science data return.

\section{Conclusion}

Figure 1 shows the expected Signal-to-Noise Ratio (SNR) of the detection of the Jupiter quadrupole deflection measurement as function of NSL initial angles $\nu_{0}$ and $\Omega_{0}$. An actual star catalogue was used to predict close encounters between bright stars and Jupiter and to accumulate the mission-total observable signal. It follows that $\nu_{0}$ is the most important initial angle to increase the detection significance: up to $8 \sigma$ detections can be reached for good choices of $\nu_{0}$. Poor choices for $\nu_{0}$ exist too (for instance around $225^{\circ}$ ) which only return $1 \sigma$ detections. We, therefore, propose to start Gaia's NSL with optimum initial conditions (e.g., $\nu_{0} \approx 341^{\circ}$ ) such that the quadrupole SNR is optimised.

Performing relativistic tests using a non-dedicated space mission has significant limitations, of both technical and programmatic nature, for instance related to ground-station scheduling and ground-station conflicts with other missions. We are currently performing a system-level trade-off analysis to reveal the (in)compatibilities of the various constraints and will report on the outcome of this study elsewhere.

\section{References}

Crosta, M. T. \& Mignard, F. 2006, Classical and Quantum Gravity, 23, 4853-4871 